\title{
Does the Screw Grip Affect the Outcome of Screw Fixation of Malleolar Fractures?
}

\section{Stamatios A Papadakis ${ }^{1 *}$, Athanasios Koutroufinis ${ }^{2}$, Dimitrios Pallis ${ }^{1}$, Athanasios Badekas ${ }^{3}$, Konstantinos Kateros $^{4}$, Olga Savidou 5 and George A Macheras ${ }^{2}$}

${ }^{1} B^{\prime}$ Department of Orthopaedics, KAT General Hospital of Attica, Greece

${ }^{2} D^{\prime}$ Department of Orthopaedics, KAT General Hospital of Attica, Greece

${ }^{3}$ Department of Foot and Ankle Surgery, Metropolitan Hospital, Athens, Greece

${ }^{4} A$ ' Department of Orthopaedics, "G Gennimatas" General Hospital, Athens, Greece

${ }^{5} A^{\prime}$ Department of Orthopaedics, Medical School of Athens University, "Attikon" University Hospital, Haidari, Greece

*Corresponding author: Stamatios A Papadakis, B' Department of Orthopaedics, KAT General Hospital of Attica, 2 Nikis Street, Kifissia, 14561, Greece

\begin{abstract}
Background: Open reduction and internal fixation with plate of the fibula is the standard method of fixation in surgically treated ankle fractures. During fixation, stripping of the distal screws is common.

Methods: The study -which is the first clinical according to the literature- included 136 patients with surgical fractures of the ankle. Patients presenting any evident factors influencing porosis as well as open fractures were excluded. Fibula reduced anatomically and fixed by a $1 / 3$ tubular plate placed laterally. Proximal screws had bicortical fixation grip strength while distal screws had only unicortical. Screws with a reduced fixation grip strength during insertion were left in place. Numbers were assigned for the plate screws, placed in sequential manner from one to ten, from distal to proximal and we recorded which of them had reduced fixation grip strength during their placement. Postoperatively, patients were immobilized in a below knee plaster for 6 weeks and bone healing evaluated with $\mathrm{X}$-ray. Further evaluation was performed after 3 and 6 months postoperative.
\end{abstract}

Results: Proximal bicortical screws had satisfactory fixation grip strength. Distal unicortical screws, $\mathrm{Nr} 1, \mathrm{Nr} 2$ and $\mathrm{Nr} 3$ presented reduced fixation grip strength in $36 \%$, $63 \%$ and $21 \%$, respectively. Postoperative there were no serious complications and all fractures healed uneventfully. Delayed union occurred in 4 cases and treated by prolonged immobilization.

Conclusions: Screws with reduced fixation grip strength in the distal holes of the fibula plate do not compromise the success of the osteosynthesis. The outcome of surgery was not affected whether or not the screws have grip or not. We recommend that screws irrespective their grip should be left in place, as they don't influence the final outcome.

\section{Keywords}

Screw grip, Malleolar fractures, Screw stripping, Plate fixation

\section{Introduction}

Malleolar fractures is one of the most common ankle injury of the lower limb [1,2]. They present better results and fewer complications when are managed by open anatomical reduction and internal fixation [3]. Conservative treatment of malleolar fractures remains an option in undisplaced and stable fractures.

Standard fixation of the fibula in malleolar fractures is achieved using a one third tubular plate, placed laterally. During fixation, screw loss of tightening of the distal screws of the fibula is a common finding and is related to the specific anatomical characteristics of the distal fibula. It is also reported, through various studies, that surgical treatment of malleolar fractures provides high healing rates and present extremely low incidence of fibula non-unions [4]. The present study is focusing on the presence of screw loss of tightening during plate fixation of the fibula and is assessing the influence of screw loss of tightening on the final clinical outcome of surgically treated malleolar fractures. 


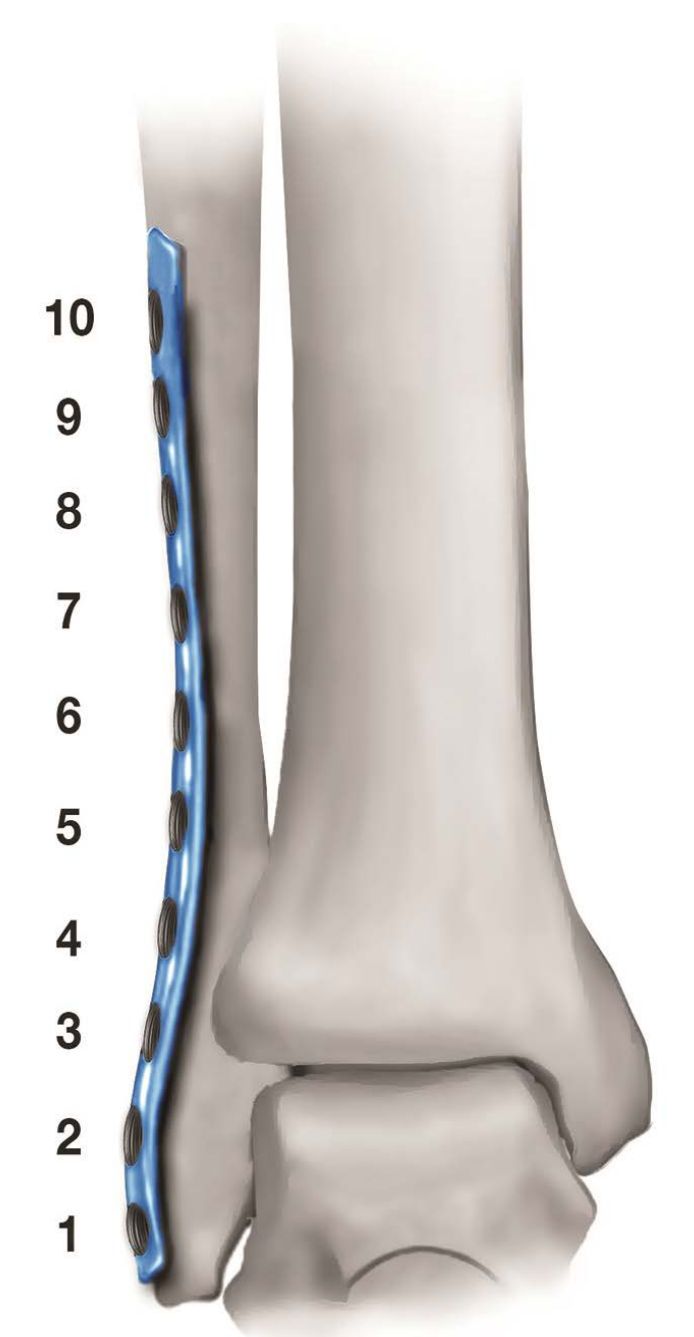

Figure 1: Schematic presentation plate placement of fibula.

\section{Materials and Methods}

One hundred and thirty-six patients suffered of malleolar fractures were treated surgically during a period of ten years (2004-2014), utilizing the AO/ASIF operative methods. The mean age of the patients was 51.7 years (range, 18-79 years). Ninety-two of the fractures were in women and forty-four in men. The majority of patients suffered the fracture after the third decade of life, with an almost equal distribution at each decade thereafter. Using the Weber classification, there were 95 (70\%) type B and 41 (30\%) type C fractures [5]. The study included all malleolar type fractures which necessitated fixation of the fibula. All patients were operated within 10 days of the fracture and were followed up for a minimum of one year. Smokers, patients with alcohol or drug abuse, diabetes, cancer, HIV, patients affected by any conditions or taking medications, with known alteration of the process of normal union, were excluded from the study. This is a descriptive study and as such no further analysis was performed. All the results were expressed as percentages.

All patients were operated by the same surgeon. The lateral approach to the fibula under tourniquet control was utilized in all cases. Fractures were fixed using the small fragment fracture instruments, standard power drill, the standard small fragment $3.5 \mathrm{~mm}$ set; $2.5 \mathrm{~mm}$ drill bit, 3.5 fully threaded cortical screws and 3.5 cancellous screws. Cancellous screws have been used to the first 45 cases and cortical screws to the rest 91 cases. The one-third tubular plate was pre-bended to fit precisely and was applied directly lateral to the distal fibula by the standard AO method. Upmost effort was taken to achieve anatomical restoration of the fibula length and rotation in all patients. Proximal screws of the fibula had bicortical fixation grip strength while distal screws were placed unicortically in order to avoid screw penetration and injury to the joint cartilage. Torque-meter screw driver was not utilized during insertion of the screws. Depending on the fracture type and characteristics, lag or syndesmotic screw were used for the fixation of the fibula and the syndesmosis. During insertion, a percentage of the distal plate screws had reduced fixation grip strength. We did not tried to replace reduced fixation grip strength screws with larger core or different type screws and decided to leave them in place. Numbers were assigned for the plate holes and the screws placed, in sequential manner from one to ten (Figure 1), from distal to proximal fibula and we recorded which of them presented reduced fixation grip strength during their placement. The medial malleolus was fixed in standard technique using two malleolar screws. Fracture reduction was evaluated intraoperatively by radiographic control with C-arm, using the tibiofibular clear space, talar tilt and mortise width and was anatomic and satisfactory in all patients.

Postoperatively, all patients were placed in a below the knee cast. No bone densitometry measurement was performed since its results do not affect the used technique. Based on our findings, the outcome was invariable and independent of the use, either cortical or cancellous screw. Moreover, our observations were mainly focused on the technique. Fracture healing was assessed clinically and radiologically. First follow up visit was in fourteen days. At six weeks patients had a new radiological evaluation and were placed in a walking cast. They were instructed to begin gradual weight bearing at eight weeks after the surgery. Our treatment protocol was to removed all syndesmotic screws after six weeks. The majority of studies advocate to remove them after 8 weeks $[6,7]$, but in this study we present our treatment protocol. At the tenth postoperative week and after consolidation of the fracture, all patients had their walking cast removed and begun ambulation with crutches for two further weeks. Criterion to our protocol for delayed union was the absent of consolidation of the fracture after three months.

\section{Results}

Plates used in most of the cases were of 7 holes $(68 / 136,50 \%)$ and 6 holes $(30 / 136,22 \%)$. In the rest of the cases, with extended comminution, longer plates, up to ten holes were used. 
Table 1: Screws utilization and stripping during insertion per plate/patient.

\begin{tabular}{|l|l|l|l|l|}
\hline Screws & Inserted/Patients & Reduced Fixation Grip Strength & Cancellous Screws & Cortical Screws \\
\hline S 1 & $136 / 136(100 \%)$ & $49 / 136(36 \%)$ & $16 / 49(33 \%)$ & $33 / 49(67 \%)$ \\
\hline S 2 & $136 / 136(100 \%)$ & $86 / 136(63 \%)$ & $28 / 86(33 \%)$ & $58 / 86(67 \%)$ \\
\hline S 3 & $58 / 136(43 \%)$ & $12 / 58(21 \%)$ & $3 / 12(25 \%)$ & $9 / 12(75 \%)$ \\
\hline Total & 330 & $147 / 330(45 \%)$ & $47 / 147(32 \%)$ & $100 / 147(68 \%)$ \\
\hline
\end{tabular}

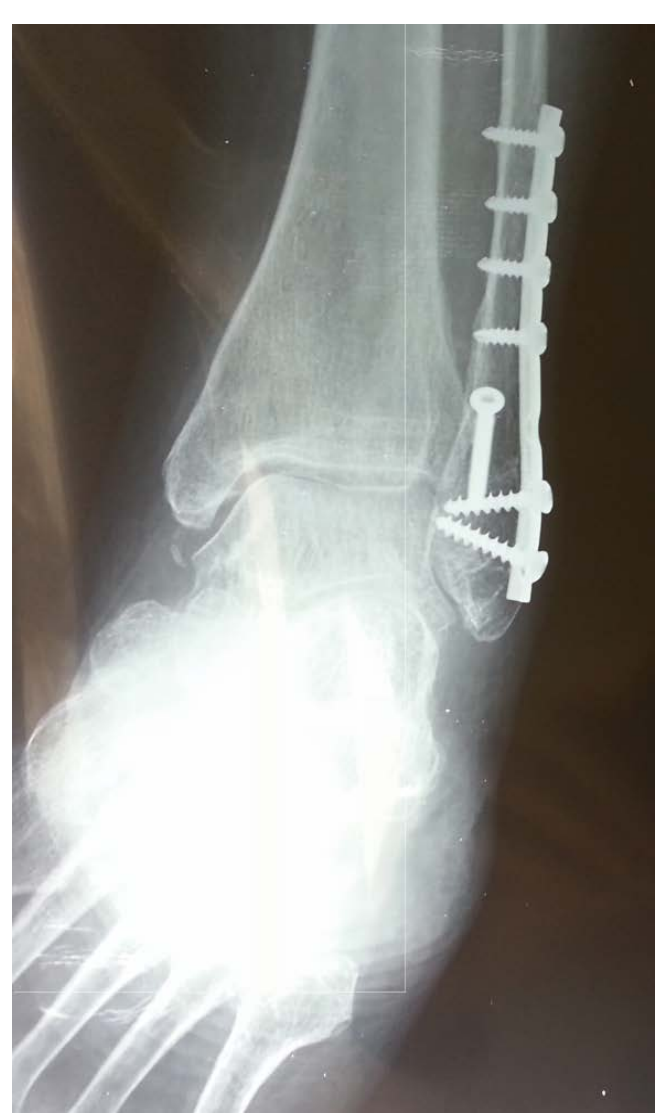

Figure 2: Post-operative AP radiograph of the ankle one month after surgery, with reduced fixation grip strength intraoperatively position 2 (S2). The reduced fixation grip is not visible.

For the fixation of the plate, screws in the first and second hole of the plates were used in all cases. The third hole screw was used only in $58(42.6 \%)$ plates, because of lag screw placement in $20(14.7 \%)$ of the fractures, syndesmotic screw placement in 7 (5.1\%) cases and finally owing to extended comminution, in the rest of the fractures.

During screw placement, proximal bicortical screws Number 4 up to the most proximal inserted screw, had satisfactory fixation grip strength. Distal unicortical screws Number 1 to Number 3 had reduced fixation grip strength in 147 (45\%) of the 330 placed screws. The first screw had reduced fixation grip strength in 49 (36\%), the second in $86(63 \%)$, and the third in $12(21 \%)$ of screws placed (Table 1). The loss of strength during screws positioning evaluated with the two fingers technique. The percentage of stripped screws increased in older patients with poor bone stock quality. However, this is not evident in intraoperative or postoperative radiographs (Figure 2).

There were no loss of reduction, no hardware failure or protrusion, no deep infection and no serious soft tissue complications. In four cases, a superficial infection was treated with antibiotics and resolved uneventfully. In four cases delayed healing occurred, but all of them healed eventually under prolonged protected immobilization. We had no cases of non-unions or pseudoarthrosis.

\section{Discussion}

Reduced fixation grip strength and screw stripping during fracture fixation is dependent on bone, screw, and plate characteristics. Bone quality is the most important factor and is represented by the bone mineral density of the trabecular and cancellous bone and by the quality and thickness of the cortical bone [8]. Specific bone anatomical characteristics and architecture may also affect the surgical technique. Poor bone quality has a detrimental effect on any orthopaedic implant regardless of the surgical technique utilized.

Screws are used for internal fixation of fractures more often than any other implant [9]. The main function of a screw is to convert the applied torque of the screwdriver during insertion, into tension along its axis while creating compression between the surfaces held together. This critical ability can be measured by (consider specific) instrumentation when testing the holding power of different types of screws in various situations. Intraoperatively the surgeon also has a rough estimate of the holding power of a screw. An indirect measurement of their fixation grip strength or holding power is the pull-out test. A screw is pulled along its longitudinal axis and the force required to rip the threads is measured. Biomechanical studies have shown that the insertion torque should not exceed $70 \%$ of the maximum torque, in order to avoid stripping of the screw [10]. Nevertheless, in clinical situations this limit is often exceeded resulting in stripped screws which may not be recognized by the surgeon intraoperatively [11].

Lateral plating of the fibula is considered the standard method of fixation in distal fibula fractures due to the easy, fast and safe approach to fibula and the ability to achieve precise restoration of length and alignment of the fibula [12]. Restoration of the anatomical length and axis of the fibula and maintenance of them is of critical importance in order to avoid disturbance of the loading forces of the ankle leading to pain and premature arthritis [13].

Anatomical studies have revealed that distal fibula bone is mainly consisting of soft cancellous bone and a thin cortex layer. The most distal part of the fibula is 
partially intraarticular, so caution has to be taken avoid intraarticular screw positioning during screw insertion and therefore unicortical placement of the distal screws is preferred. Harnroongroj, demonstrated in his experimental study on femora, that the metaphyseal cancellous bone area plays little role in creation of screw-holding force during insertion of cancellous screws [14]. A cancellous screw placed in metaphyseal zone achieves sufficient holding force only when it has bicortical engagement and has greater holding force than a unicortical inserted one. It is reported that during lateral plating screw insertion, the most distal screws often have poor fixation grip strength and can loosen, leading to loss of fixation and possible nonunion or malunion [15-18]. It is also reported that unicortical screw placement in the primarily cancellous bone of the distal fibula may lead to less than satisfactory fixation, especially when comminution, osteoporosis, or a small distal fragment is present $[16,17,19]$. Reduced fixation grip strength and stripping of at least one screw during insertion occurs in up to $88 \%$ of patients more than 50 -years-old [20]. In a biomechanical study in osteoporotic bone, $21 \%$ of the inserted screws stripped [21].

Our study, which is to our knowledge the only one in the literature based on intraoperative findings, confirmed the increased screw stripping during lateral plating of the fibula in $45 \%$ of the three distal screws (ranging from $18 \%$ to $63 \%$ ). The percentage of stripping was even higher for the older population with advanced osteoporosis.

Various solutions to this intraoperative finding have been implemented, aiming to improve screw fixation grip strength, plate and fracture stabilization, achieve stable fixation for early mobilization and avoid possible complications as malunion or nonunion of the fracture. The utilization of untapped partial threaded cancellous screws or cortical untapped screws is recommended by the AO group [12]. The locking plate has several advantages in severely osteoporotic bone, does not require bicortical screw placement and is not dependent of the distal fibula bone mineral density [22]. The posterior placed antiglide plate that allows bicortical screw insertion with reduced risk of intraarticular penetration is also a frequently used alternative to the lateral plate. The cadaveric study by Schaffer and Manoli concluded that the posterior-antiglide plate has more torsional stability than the lateral placed plate fixed with unicortical screws. It is also known though, that the posterior plating of the fibula requires more dissection and stripping of the fracture and poses a greater danger of peroneal tendon injury. Special screw placement techniques that allow bicortical screw placement during lateral plating without the risk of intraarticular placement [23], Kirshner wires and intramedullary fibula nails are also used to aid fixation. Nylon cavity plugs and augmentation with polymethylmethacrylate bone cement, calcium phosphate cement or hydroxyapatite composite resin for fixation have been advocated as enhancers of fixation grip strength of screws in poorly mineralized bone. Local application of drugs as biphosphonates on the screw hole has been tested as a method to reduce the bone catabolism in the screw-bone interface and therefore improve anchorage.

It is considered that if the screws cannot create and maintain tension and compression between the plate and bone, this may impair the fit of the plate to the bone, the safety of the reduction, the reduction and compression between the bone fragments and may result in delayed or impaired union, malunion or nonunion. In distal fibula fractures fixed by lateral plating, the danger of loss of reduction, length and rotation seems imminent with such a high percentage of screws with reduced fixation grip strength. In our study this intraoperative finding though, did not affect the quality of the reduction after the initiation of partial weight bearing. It didn't affect the time of union or the final result either. We had no hardware related complications and all fractures healed uneventfully. Our findings of extremely low nonunion rate coincide with the study of Mendelsohn which reviewed 253 patients with ankle fractures over a 10-year period and noted nonunion of the lateral malleolus in 2 patients (1.1\%) [24] and Beris, et al. which reviewed 144 patients with malleolar fractures over the same period of time and recorded one nonunion of the lateral malleolus which healed uneventfully after revision surgery [25].

\section{Acknowledgements}

Not applicable.

\section{Conflicts of Interest}

The authors declare that they have no conflicts of interest.

\section{References}

1. Salai M, Dudkiewicz I, Novikov I, Amit Y, Chechick A (2000) The epidemic of ankle fractures in the elderly- Is surgical treatment warranted? Arch Orthop Trauma Surg 120: 511513.

2. Lamontagne J, Blachut PA, Broekhuyse HM, O'Brien PJ, Meek RN (2002) Surgical treatment of a displaced lateral malleolus fracture: The antiglide technique versus lateral plate fixation. J Orthop Trauma 16: 498-502.

3. White NJ, Corr DT, Wagg JP, Lorincz C, Buckley RE (2013) Locked plate fixation of the comminuted distal fibula: A biomechanical study. Can J Surg 56: 35-40.

4. Leyes M, Torres R, Guillen P (2003) Complications of open reduction and internal fixation of ankle fractures. Foot Ankle Clin 8: 131-147.

5. Weber BG (1972) Die Verletzungen des oberen Sprunggelenkes. ( $2^{\text {nd }}$ edn), Verlag Hans Huber, Berne, Switzerland.

6. Geissler WB, Tsao AK, Hughes JL (1996) Fractures and injuries of the ankle. In: Rockwood Jr CA, Green DP, Bucholz RW, Rockwood and Green's fractures in adults. ( $4^{\text {th }}$ edn), Lippincott-Raven, Philadelphia, 2242-2244. 
7. Van den Bekerom MP, Hogervorst M, Bolhuis HW, Van Dijk CN (2008) Operative aspects of the syndesmotic screw: Review of current concepts. Injury 39: 491-498.

8. Litchfield JC (1987) The treatment of unstable fractures of the ankle in the elderly. Injury 18: 128-132.

9. Perren S, Cordey J, Baumgart F (1992) Technical and biomechanical aspects of screws used for bone surgery. Int J Orthop Trauma 2: 31-48.

10. Cleek TM, Reynolds KJ, Hearn TC (2007) Effect of screw torque level on cortical bone pullout strength. J Orthop Trauma 21: 117-123.

11. Cordey J, Rahn BA, Perren SM (1980) Human torque control in the use of bone screws. In: Uhthoff HK, Stahl E, Current Concepts of Internal Fixation of Fractures. Springer-Verlag, New York, 235-243.

12. Haidukewych GJ (2004) Innovations in locking plate technology. J Am Acad Orthop Surg 12: 205-212.

13. Rukavina A (1998) The role of fibular length and the width of the ankle mortise in post-traumatic osteoarthrosis after malleolar fracture. Int Orthop 22: 357-360.

14. Harnroongroj T, Techataweewan A (1999) Determination of the role of the cancellous bone in generation of screw holding power at metaphysis. Clin Biomech (Bristol Avon) 14: 364-366.

15. Hahn D, Colton C (2000) Malleolar fractures. In: Principles of fracture management. Thieme, 559-581.

16. Beauchamp CG, Clay NK, Thexton PW (1983) Displaced ankle fractures in patients over 50 years of age. J Bone Joint Surg Br 65: 329-332.
17. Broos PL, Bisschop AP (1991) Operative treatment of ankle fractures in adults: Correlation between types of fracture and final result. Injury 22: 403-406.

18. Brunner CF, Weber BG (1982) The antiglide plate. In: Special Techniques in Internal Fixation. Springer - Verlag, New York, 115-133.

19. Guille JT, Lipton GE, Bowen JR, Uthaman U (1997) Delayed union following stress fracture of the distal fibula secondary to rotational malunion of lateral malleolar fracture. Am J Orthop 26: 442-445.

20. Andreassen GS, Hoiness PR, Skraamm I, Granlund O, Engebretsen $L$ (2004) Use of a synthetic bone void filler to augment screws in osteopenicankle fracture fixation. Arch Orthop Trauma Surg 124: 161-165.

21. Dinah F, Mears SC, Knight TA, Soin SP, Campbell JT, et al. (2011) Inadvertent screw stripping during ankle fracture fixation in elderly bone. Geriatr Orthop Surg Rehabil 2: 86-89.

22. Kim T, Ayturk U, Haskell A, Miclau T, Puttlitz CM (2007) Fixation of osteoporotic distal fibula fractures: A biomechanical comparison of locking versus conventional plates. J Foot Ankle Surg 46: 2-6.

23. Milner BF, Mercer D, Firoozbakhsh K, Larsen K, DeCoster TA, et al. (2007) Bicortical screw fixation of distal fibula fractures with a lateral plate: An anatomic and biomechanical study of a new technique. J Foot Ankle Surg 46: 341-347.

24. Mendelsohn HA (1965) Non-union of malleolar fractures of the ankle. Clin Orthop Relat Res 42: 103-120.

25. Beris AE, Kabbani KT, Xenakis TA, Mitsionis G, Soucacos PK, et al. (1997) Surgical treatment of malleolar fractures. A review of 144 patients. Clin Orthop Relat Res: 90-98. 